\title{
High Efficiency Street Lightning using ZigBee based Online Control System
}

\author{
S. Anu Thilak ${ }^{1}$, P. Narmadha ${ }^{2}$, G. Yoga $^{3}$, B. Vijayalakshmi $^{4}$ \\ M.E, Saranathan College of Engineering, Trichy, India ${ }^{1,2,3}$ \\ Assistant Professor, EEE, Saranathan College of Engineering Trichy, India ${ }^{4}$
}

\begin{abstract}
This paper proposed online control system can optimize management and efficiency of street lighting systems. It uses ZigBee based wireless devices which enable more efficient street lamp-system management. In this work an advanced interface and control architecture are used. It uses a sensor based ZigBee combination to control and guarantee the desired system parameters. The information is transferred point by point using ZigBee transmitters and receivers and is sent to a control terminal used to check the state of the street lamps and to take appropriate measures in case of failure.
\end{abstract}

Keywords: Automation, ZigBee network, sensor, emergency devices, control units.

\section{INTRODUCTION}

Lighting systems, especially in the public sector, are still designed according to the old standards of reliability and they often do not take advantage of the latest technological developments. In many cases, this is related to the plant administrators who have not completed the return of the expenses derived from the construction of existing facilities yet. However, the recent increasing pressure related to the raw material costs and the greater social sensitivity to environmental issues are leading manufacturers to develop new techniques and technologies which allow significant cost savings and a greater respect for the environment [1-3]. The first possible solution is the use of new technologies for the sources of light. In this area, light- emitting diode (LED) technology is the best solution because it offers many benefits. Researchers have already considered this possibility, designing an advanced street lighting system based on LEDs. The second possible solution, and perhaps the most revolutionary, is the use of a remote-control system based on intelligent lamp posts that send information to a central control system, thus simplifying management and maintenance issues. The control is implemented through a network of sensors to collect the relevant information related to the management and maintenance of the system, transferring the information via wireless using the ZigBee protocol. The field of the ZigBee remote sensing and control system is widely present in the literature. The ZigBee systems similar to the lighting systems in structure and management. In this paper, the system is able to integrate the latest technologies, in order to describe an advanced and intelligent management and control system of the street lighting.

\section{DEVICES AND METHODS}

Fig1.shows the conceptual scheme of the proposed system. It consists of a group of observation stations on the street (one station for each lamp post) and a base station typically placed in a building located nearby. It is a modular system, easily extendable. The measuring stations monitor the street conditions and the intensity of sunlight and, based on them, they decide to turn the lamps ON or OFF.

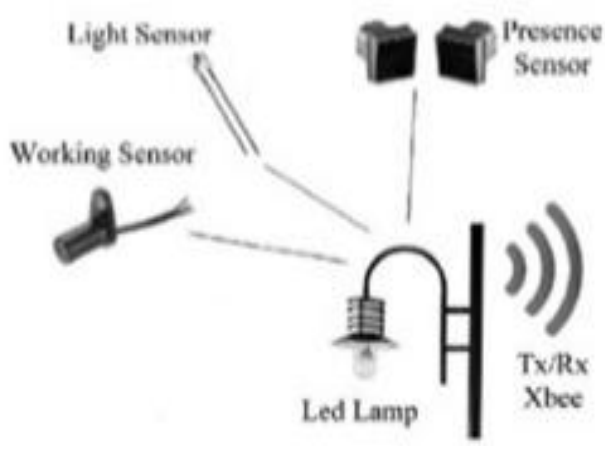

Fig 1 Components

The conditions depend on the pattern of the street where the lights are located and on the solar irradiation at a given point of the street, with frequent changes, depending on weather conditions, season, geographical location, and many other factors. For these reasons, each lamp completely independent in the management of its own lighting. The on-street station also checks if the lamp is properly working and sends the information through the wireless network to the base station for processing data. Fig 2 and Fig 3 shows the transmitter and receiver section respectively.

The monitoring station located in each lamp post consists of several modules: the light sensor, the failure sensor, and an emergency switch. These devices work together and transfer all of the information to a microcontroller which processes the data and automatically sets the appropriate course of action. 
Vol. 6, Issue 1, January 2017

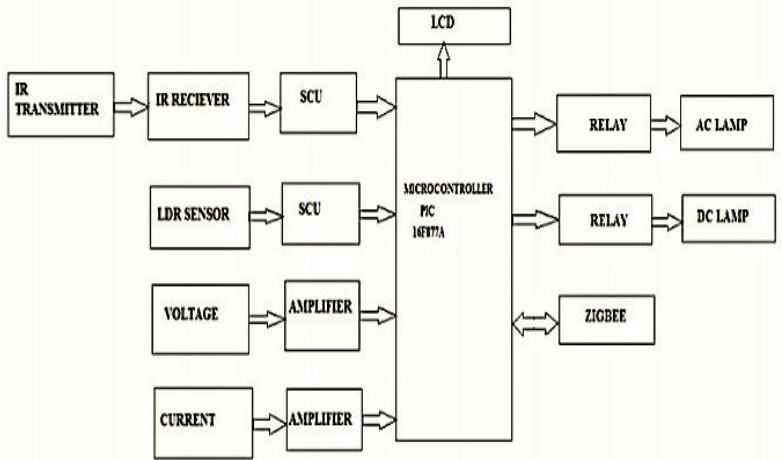

Fig 2 Transmission Section

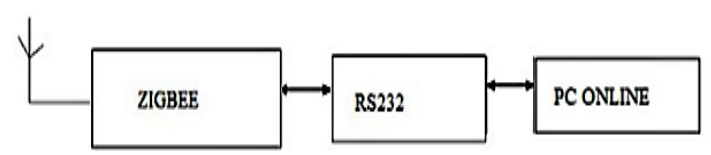

Fig 3. Receiver Section

A priority in the transmission of information is assigned to each sensor, for example, the emergency switch takes precedence over any other devices.

\section{HARDWARE DESCRIPTION}

\section{A. Presence Sensor}

The task of the presence sensor is to identify the passage of vehicle or pedestrian, giving an input to turn ON the lamp or a group of lamps. This function depends on the pattern of the street, in case of the street without cross roads, a single sensor is sufficient (or one at each end in case of a two-way street), while for a street requiring more precise control, a solution with multiple presence detectors is necessary.
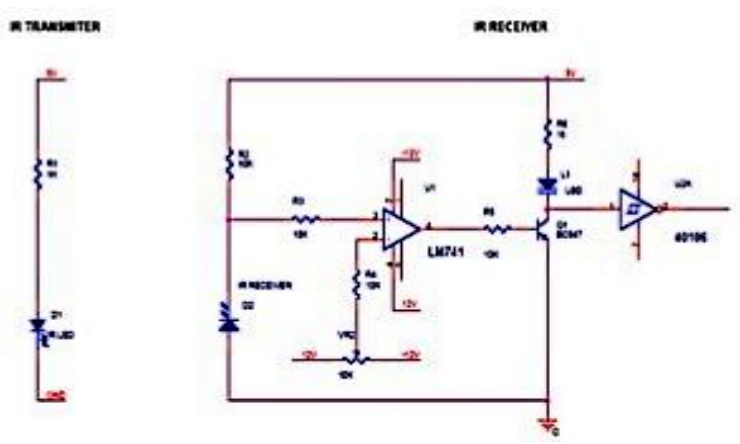

Fig 4. IR Transmitter and Receiver

Infrared transmitter is one type of LED which emits infrared rays generally called as IR Transmitter. Similarly, IR Receiver is used to receive the IR rays transmitted by the IR transmitter. One important point is both IR transmitter and receiver should be placed straight line to each other. The transmitted signal is given to IR transmitter whenever the signal is high, the IR transmitter LED is conducting it passes the IR rays to the receiver. The IR receiver is connected with comparator. The comparator is constructed with LM 741 operational amplifier.

\section{B. Light Sensor \\ LDR Controlled illumination controller}

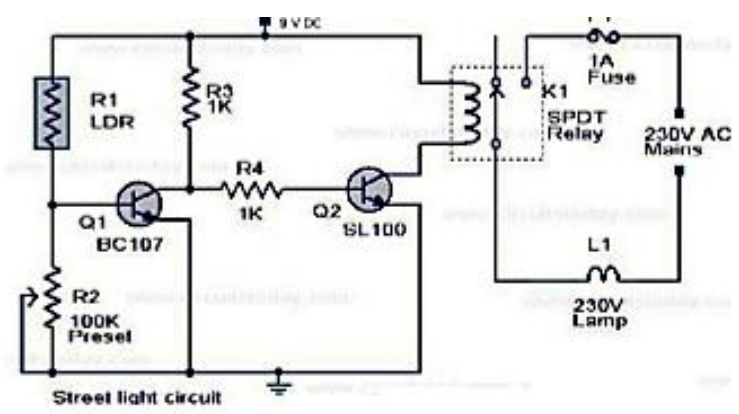

Fig 5. LDR Sensor

LDR sensor is shown in Fig 5. The LDR light dependent resistor is varying resistance with light intensity. This will be mostly linear to the light intensity. During the darkness, the resistance of LDR shoots up to Meg ohm ranges. When LDR is illuminated by means of the sunlight, the resistance of LDR suddenly decreases (below 10 kilo $\mathrm{ohm})$. Then the LDR is connected to a wheat stone bridge. There the resistance is converted into differential voltage, then the voltage is amplified and given to the ADC. The amplifier will give the output voltage corresponds to the light intensity with the required level and it will produce output depends on the intensity. This $\mathrm{O} / \mathrm{P}$ is connected to the PC through an ADC.

\section{CONTROL STRATEGIES}

A light sensor can measure the brightness of the sunlight and provides information [6-8]. The purpose of this measurement is to ensure a minimum level of illumination of the street. The sensor must have high sensitivity in the visible spectrum, providing a photocurrent high enough for low light luminance levels. For this reason, the phototransistor TEPT5700 (by Vishay Semiconductors) has been selected. Based on the measured luminance, the microcontroller drives the lamp in order to maintain a constant level of illumination. This action is obviously not required during daylight time, but it is desirable in the early morning and at dusk, when it is not necessary to operate the lamp at full power but simply as a "sup-port" to the sunlight. This mode enables saving electric power supplied to the lamp because the lamp is regulated by the combined action of the sensor and the microcontroller to ensure the minimum illumination required.

\section{Working Sensor}

Voltage measurement

This circuit is designed to monitor the supply voltage. The supply voltage that has to monitor is step down by the potential transformer. Usually we are using the $0-6 \mathrm{~V}$ potential transformer. The step-down voltage is rectified by the precision rectifier. The precision rectifier is a configuration obtained with an operational amplifier in order to have a circuit behaving like an ideal diode or rectifier. 
Vol. 6, Issue 1, January 2017

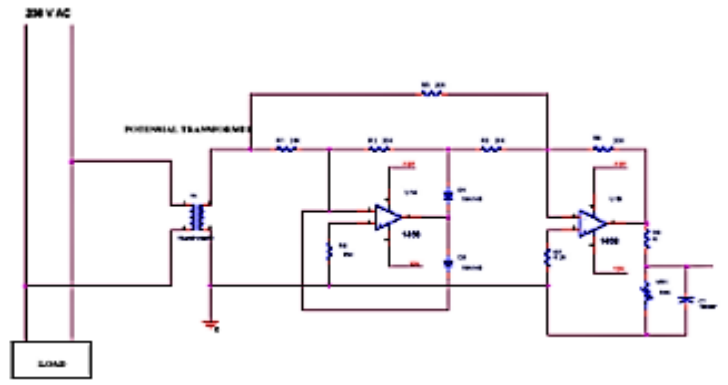

Fig 6. Voltage Measurement

Current measurement

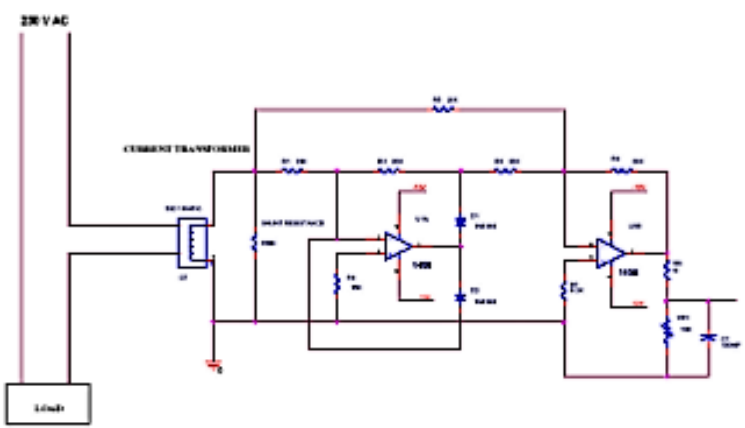

Fig 7. Current Measurement

Fig 7. Current measurement circuit is designed to monitor the supply current. The supply current that has to monitor is step down by the current transformer. The step-down current is converted by the voltage with the help of shunt resistor. Then the converted voltage is rectified by the precision rectifier. The precision rectifier a configuration obtained with an operational amplifier in order to have a circuit behaving like an ideal diode or rectifier.

\section{BASE CONTROL STATION}

The base control station is the hub of the system since it allows the visualization of the entire lighting system. The transmission system consists of a ZigBee device that receives information on the state of the lamps and sends it to a terminal. The processing unit consists of a terminal with a serial Universal Asynchronous ReceiverTransmitter (UART) interface which receives information about the state of the lamps provided by a ZigBee device.

The terminal is required for a graphical display of the results. Moreover, data on lamp's operation are associated with the lamp address; consequently, all faults are easily identified. The graphical interface enables monitoring the state of the system with the state of the lights and the power consumption of each lamp. The operator will have a graphical representation of the lamp location within the area where the system is installed.

\section{ZIGBEE NETWORK}

ZigBee is a wireless communication technology based on the IEEE802.15.4 standard for communication among multiple devices in a wireless personal area network
(WPAN) [9-12]. The typical distance of a ZigBee transmission range, depending on the environment conditions and the transmission power, shifts from tens to hundreds of meters, and the transmission power is deliberately kept as low as possible (in the order of a few milli watts) to maintain the lowest energy consumption. In the proposed system, the network is built to transfer information from the lamp posts to the base station control. Information is transferred point by point, from one lamp post to another where each lamp post has a unique address in the system. Each lamp post can only send the information to the nearest one, until the information reaches the base station.

Thus, transmission power is limited to the required low value and the signals sent by the lamp posts do not interfere with each other. In case of failure of one lamp, the chosen transmission distance between the lamp posts ensures that the signal can reach the next operational lamp post without breaking the chain. The ZigBee wireless communication network has been implemented with the use of Digi-MaxStream radio-frequency modules called XBee modules, which are available in Standard and Pro versions (pin-top in compatible). The Standard Xbee modules have an operation range of tens of meters indoors and hundreds of meters outdoors, while the XBee Pro modules have a wider spread range in the order of hundreds of meters indoors and of about $1.5 \mathrm{Km}$ outdoors, because the Pro modules have higher transmission power, but imply higher consumption (about three times the consumption of the Standard version). The receiver has very high sensitivity and a low probability of receiving corrupted packets (less than 1\%).

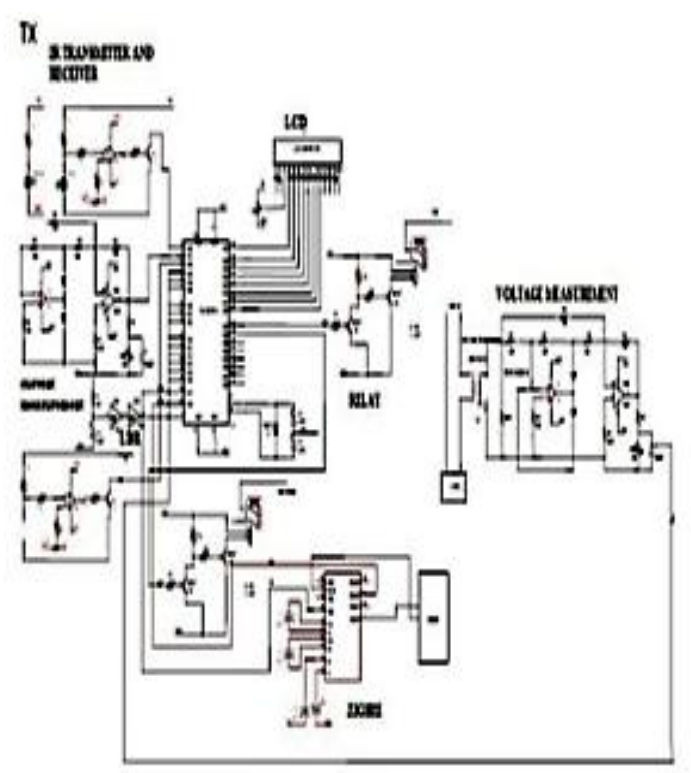

Fig 8. Transmission Section

The simulation model of transmitter and receiver sections is given in Fig 8 \& Fig 9 respectively. The Hardware circuit of the above simulation model is also depicted in Fig 10 and Fig 11. 
Vol. 6, Issue 1, January 2017

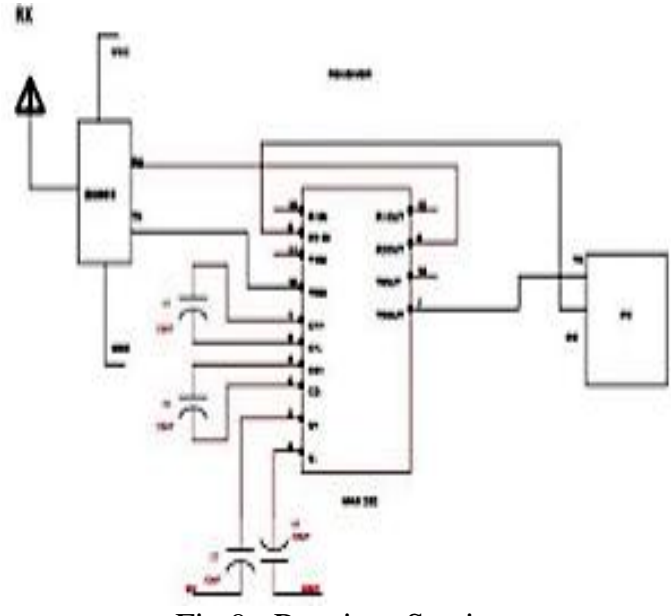

Fig 9. Receiver Section

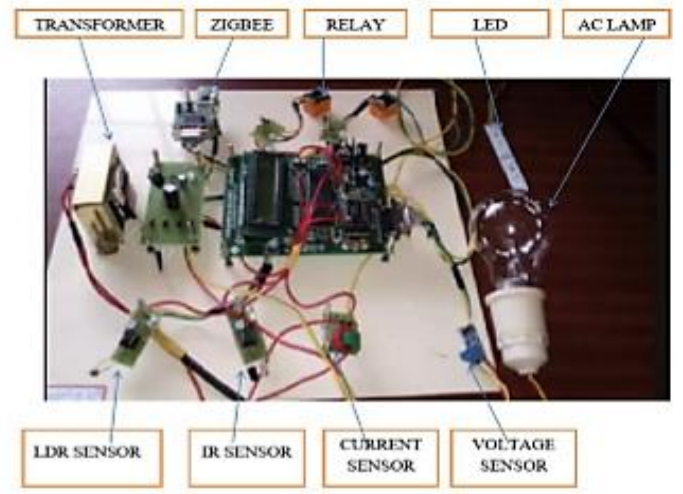

Fig 10 Transmission Circuit

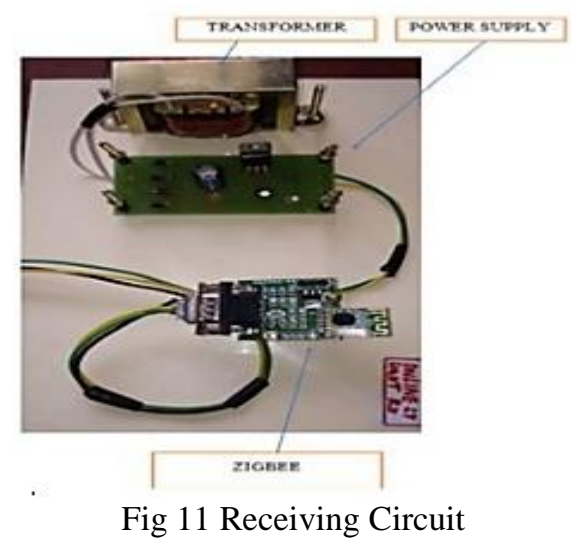

VI. CONCLUSION

This paper describes a new intelligent street lighting system which integrates new technologies available on the market to offer higher efficiency and considerable savings. This can be achieved using the highly efficient LED technology supplied by renewable energy of solar panels, for which the cost of energy is independent from the power supplier prices, combined to an intelligent management of the lamp posts derived by a control system switching on the light only when necessary, increasing the lamps' lifetime. Another advantage obtained by the control system is the intelligent management of the lamp posts by sending data to a central station by ZigBee wireless communication. The system maintenance can be easily and efficiently planned from the central station, allowing additional savings. The proposed system is particularly suitable for street lighting in urban and rural areas where the traffic is low at a given range of time. The independent nature of the power supply network enables implementing the system in remote areas where the classical installations are prohibitively expensive. The system is always flexible, extendable, and fully adaptable to user needs. The simplicity of ZigBee, the reliability of electronic components, the feature of the sensor network, the processing speed, the reduced costs, and the ease of installation are the features that characterize the proposed system, which presents itself as an interesting engineering and commercial solution as the comparison with other technologies demonstrated. The system can be adopted in the future for loads supplied by the power system, which enables the monitoring of energy consumption. This situation is particularly interesting in the case of economic incentives offered to clients that enable remote control of their loads and can be useful, for example, to prevent the system blackout. Moreover, new perspectives in billing and in the intelligent management of remotely controlled loads and for smart grid and smart metering applications.

\section{FUTURE SCOPE}

The same project can be implemented by using solar energy instead of transmission system [4]. This will reduce the overall running cost. This type of online street light control will be more efficient as the solar energy plays a major role in future. Though the initial cost is increased, it can be reduced by the overall running cost.

\section{REFERENCES}

[1] Caponetto.R, Dongola, Fortuna, Rician, and Zufacchi.D (2008) "Power consumption reduction in a remote-controlled street lighting system"

[2] Chen P.Y, Liu.Y.H, Yau. Y.T, and Lee H.C (2008) "Development of an energy efficient street light driving system"

[3] Chen Y and Liu.Z (2009) "Distributed intelligent city street lamp monitoring and control system based on wireless communication chip nRF401"

[4] Costa.M.A.D, Costa.G.H, Dos Santos.A.S, Schuch.L, and Pinheiro.J.R (2009), "A high efficiency autonomous street lighting system based on solar energy and LEDs"

[5] Yongqing.W, Chuncheng.H, SuoliangZ, Yali.H and Hong.W (2009) "Design of solar LED street lamp automatic control circuit"

[6] Yue.W, Changhong.S, Xianghong.Z, and Wei.Y (2010) "Design of new intelligent street light control system"

[7] D.Gislason, Zigbee Wireless Networking, 1st ed. Burlington, MA: Newness, 2008.

[8] "Product Manual v1.xAx - 802.15.4 Protocol for OEM RF Module Part Numbers: XB24-...-001, IEEE 802.15.4 OEMRF Modules by Maxstream," Maxstream, Inc., London, UT, 2007. [Online]. Available: ftp://ftp1.digi.com/support/documentation/manualboe mrfmodules802.15.4v1.xAx.pdf

[9] D. Gislason, "Zigbee applications-Part3: ZigBee PANs," 2010. [Online].

[10] Zigbee Alliance, ZigBee specification overview. 2012. [Online] Available: http://www.zigbee.org/Specifications/ZigBee/Over view. aspx

[11] Ente Italiano di Unificazione (UNI), Riscaldamento eraffrescamen todegliedifici.Dataclimatici.1994[Online].Available:http://re.jrc.ec. europa.eu/pvgis/

[12] Harbin Hopeful Star Co., "CMP12 datasheet," no. 3, 2012.[Online] Available: http://re.jrc.ec.europa.eu/pvgis/. 